\title{
Golden plaster for pain therapy in patients with knee osteoarthritis: study protocol for a multicenter randomized, double-blind, placebo-controlled trial
}

\author{
Jin-Tao Liu ${ }^{1,2}$, De-Zhi Tang ${ }^{2 *}$, Xiao-Feng Li ${ }^{2}$, Zhi-Gang Zhang ${ }^{1}$, Wan-Bo $\mathrm{Ji}^{3}$, Shuai Tao ${ }^{4}$, Yong-Jun Wang ${ }^{2}$ \\ and Hong Jiang ${ }^{1 *}$
}

\begin{abstract}
Background: Osteoarthritis is a relatively common musculoskeletal disorder that increases in prevalence with age. Worldwide, knee osteoarthritis is one of the leading causes of disability, particularly in the elderly. In numerous trials of agents for long-term pain therapy, no well-established and replicable results have been achieved. Complementary and alternative medical approaches have been employed for thousands of years to relieve knee osteoarthritis pain. Among herbal medicines, the golden plaster is the preferred and most commonlyused method in China to reduce pain in patients with knee osteoarthritis, as it causes few adverse effects. The purpose of this study will be to evaluate the efficacy and safety of golden plaster on pain in patients with knee osteoarthritis.

Methods/Design: This study will be a multicenter randomized, double-blind, placebo-controlled trial. A total of 320 participants aged 45 to 79 years with knee osteoarthritis, whose scores on a visual analog scale (VAS) are more than $20 \mathrm{~mm}$, will be randomly allocated into a treatment group and a control group. A golden plaster will be administered externally to participants in the treatment group for 2 weeks, while the control group will receive a placebo plaster externally for 2 weeks. Follow-up will be at regular intervals during a 4-week period with a VAS score for pain, quality of life, and complications.
\end{abstract}

Discussion: This study will be a methodologically sound randomized controlled trial to assess pain relief after the intervention of golden plaster, compared to a placebo intervention in patients with knee osteoarthritis.

Trial registration: ClinicalTrials.gov identifier: ChiCTR-TRC-13003418

Keywords: Clinical trials, Golden plaster, Knee osteoarthritis, Visual analog scale

\section{Background}

Osteoarthritis (OA), a progressive joint disorder, is a common health issue in the aging population worldwide [1,2]. The prevalence rate of OA is $28 \%$ in people over 45 years old and $37 \%$ in people over 65 years old [3]; thus, its prevalence and health impact increase with age. Knee OAis one of the leading causes of disability, particularly in the elderly. The aim of OA treatmentisto reduce or control

\footnotetext{
* Correspondence: dztang702@126.com; doctorhong@yeah.net

${ }^{2}$ Longhua Hospital, Shanghai University of Traditional Chinese Medicine, 725

Wan-Ping South Road, Shanghai 200032, PR China

'Suzhou Hospital of Traditional Chinese Medicine, 889 Wuzhongxi Road,

Suzhou, Jiangsu 215009, PR China

Full list of author information is available at the end of the article
}

pain, improve physical function, prevent disability, and enhance quality of life. The non-surgical management of $\mathrm{OA}$, namely pharmacologic treatment combined with physical therapy, is utilized more and more widely $[4,5]$. Pharmacologic treatments include oral analgesics, acetaminophen, ibuprofen, and traditional Chinese medicinal (TCM) products [6,7]. Several systematic reviews have outlined and showed the effectiveness of muscle strengthening or aerobic exercises in the osteoarthritic population [8-11]. Acupuncture and Tuina, two kinds of the physical therapy, have also been used for the management of OA [6,7]. Despite numerous trials, investigators have not been able to establish replicable relief of long-term pain.

\section{Biomed Central}

(c) 2013 Liu et al.; licensee BioMed Central Ltd. This is an open access article distributed under the terms of the Creative Commons Attribution License (http://creativecommons.org/licenses/by/2.0), which permits unrestricted use, distribution, and reproduction in any medium, provided the original work is properly cited. 
Complementary and alternative medicine has been employed over thousands of years to relieve knee OA pain. Plasters are a very important therapeutic method in TCM, inducing muscle relaxation, invigorating blood circulation, and improving electrolyte balance and bile release [12,13]. Among herbal medicines, the golden plaster is the preferred and most commonly used method in China for pain reduction in patients with knee OA, as they cause very few adverse effects. Due to the absence of a gold standard for the treatment of knee $\mathrm{OA}$, this trial will be conducted to detect the effectiveness of these herbal medicines compared to a placebocontrolled group. Therefore, we designed a randomized, double-blind, placebo-controlled trial of golden plaster in patients with knee OA.

\section{Methods/Design}

\section{Study design}

This clinical trial will be a multicenter, randomized, double-blind, placebo-controlled trial. Subjects will be enrolled at four hospitals: i) Suzhou Hospital of TCM; ii) Longhua Hospital affiliated to Shanghai University of TCM; iii) Zhangjiagang Hospital of TCM; and iv) Taizhou Hospital of TCM.

The Ethics Boards of the Suzhou Hospital of TCM, Longhua Hospital affiliated to Shanghai University of TCM, Zhangjiagang Hospital of TCM, and Taizhou Hospital of TCM have approved this study. Each participating center has obtained local Institutional Review Board approval. All study participants will sign the written informed consent before participation.

\section{Inclusion criteria}

The inclusion criteria are as follows: age 45 to 79 years; symptomatic knee OA with a pain of at least $20 \mathrm{~mm}$ on a $100-\mathrm{mm}$ visual analog scale (VAS); American College of Rheumatology criteria for symptomatic knee OA assessed by a rheumatologist [14]; ability to read, speak, and understand English or Chinese; and capability of understanding the study requirements and willingness to cooperate with the study instructions.

\section{Exclusion criteria}

Exclusion criteria are as follows: psoriatic arthritis; lupus or cancer; severe cardiac or renal impairment; significant trauma to knees, including arthroscopy or significant injury to ligaments or the menisci of the knee within one year preceding the study; allergy to plaster.

\section{Recruitment}

Participants will be recruited through advertisements in local newspapers, bulletin boards, and on the websites of local medical centers. All patients will be screened initially by baseline assessment with regard to selection criteria before randomization. If inclusion criteria are met and the informed consent form is signed, the patient will be sent to randomization.

\section{Randomization}

The teletherapist, a research nurse, will then register the participant into the database for randomization. If the participant is ready to be randomized, the teletherapist enters "yes"; the site-specific randomization program behind the form displays the participant's group assignment number (placebo versus golden plaster). Sitespecific randomization lists will be computer-generated (i.e., generated by an individualized basic visual code program) and concealed from the researchers by a senior data manager who is not involved in the study. This information will remain confidential and is not shared with the study sites, in accordance with the CONSORT guidelines. This trial uses a prospective, randomized, outcome-blinded design, in which all outcome assessments are made by a research assistant blinded to treatment allocation and uninvolved in patient consent and management.

\section{Intervention}

This study will be conducted in accordance with the requirements outlined in the Declaration of Helsinki, and approved by the appropriate Institutional Review Boards. Each participant will sign the written informed consent form before undergoing any examination or study procedure, in compliance with Good Clinical Practice. Eligible patients will be randomized into one of the two groups: placebo and golden plaster. All drugs will be administered externally for 2 weeks. Participants are given in-person instructions for using the plaster by our research nurses, who will be trained before this study. The trial will be double-blind: the treating physicians, subjects, investigators and statisticians are unaware of treatment assignment. Randomization of subjects will occur centrally using a random number generator. Patientvisitswill be performed at base line, and 1-, 2-, 3-, and 4-week follow-up after treatment (Figure 1). Assessments will be made during all five visits. Used plasters will be reclaimed at the 1- or 2-week follow-up visit.

The investigational group will receive one medicated plaster, to be used twice a day (morning and evening, approximately every 12 hours and at the same time each day) for 14 consecutive days. The golden plaster is a $10 \times 15 \mathrm{~cm}$ medicated adhesive patch, composed of Trichosanthes root, turmeric, angelica, Atractylodes, licorice, rhubarb, Phellodendron, Arisaema, Magnolia bark, tangerine peel, sesame oil, Huang Dan, and a hydrophilic adhesive vehicle.

The control group will receive placebo plasters (containing the hydrophilic adhesive vehicle only), which are 


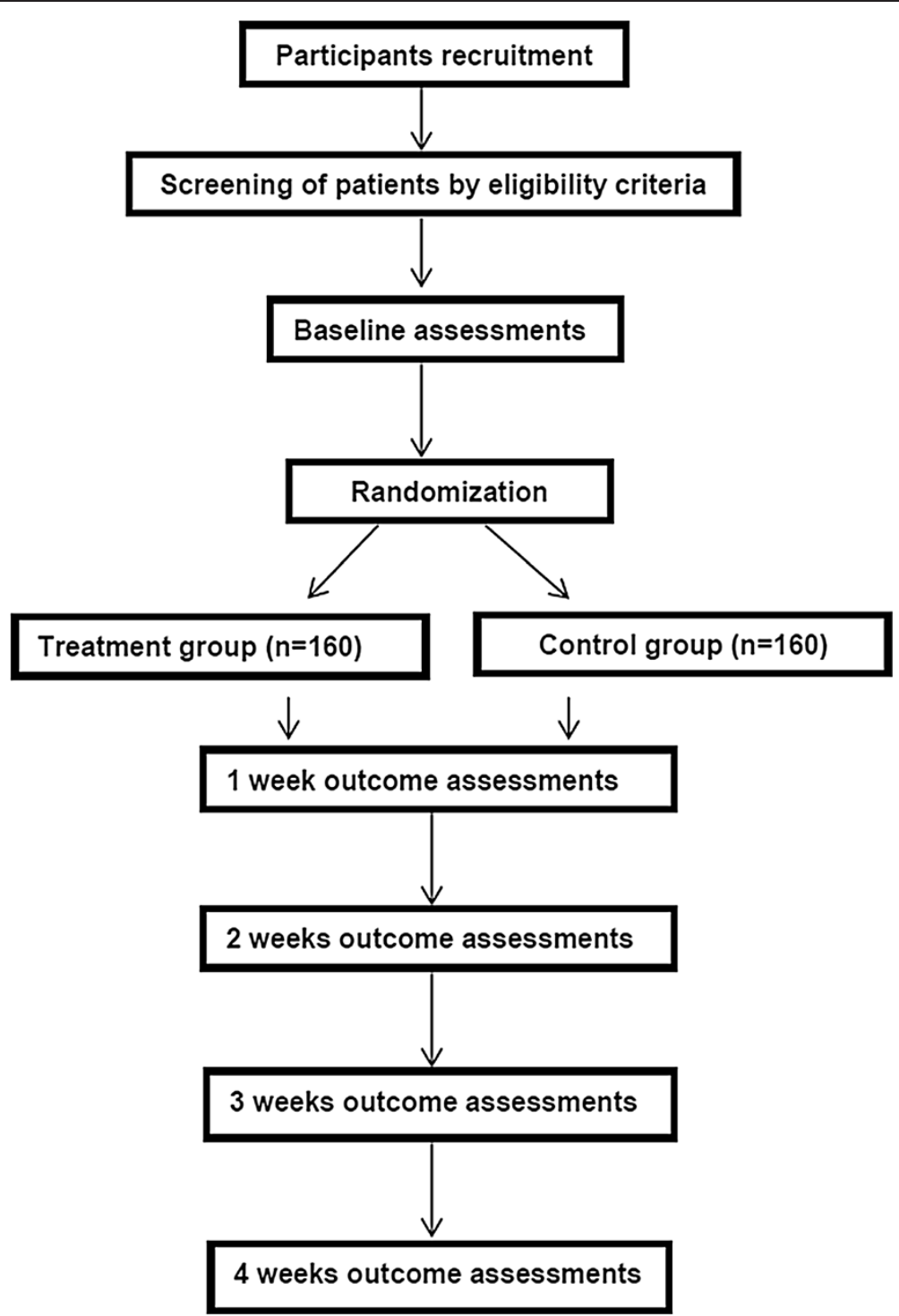

Figure 1 Study flow diagram, including recruitment, eligibility, screening, randomization, and outcome assessments.

identical to the tested formulation in terms of texture, size, color, and odor. The tested products will be packed and labeled, according to current Good Manufacturing Practice and legal requirements, by the Suzhou Hospital of TCM to ensure the participants and all personnel involved in the study will remain fully blinded as to the identity of the treatment administered. During the process, patients will not be allowed to use other drugs. If they do so, patients are required to provide feedback to the investigators and discontinue involvement in the study.

\section{Outcome measures}

Primary outcome measure

The primary efficacy endpoint of the study will be the VAS, which is a pain score ranging from $0 \mathrm{~mm}$ (no pain) to $100 \mathrm{~mm}$ (worst pain ever experienced) [15]. Operationally, the VAS score is usually a horizontal line, $100 \mathrm{~mm}$ in length, anchored by word descriptors at each end. Patients mark the point on the line of current pain. The VAS score is then determined by measuring in millimeters from the left hand end of the line to the point that the patient marked. The VAS score will be measured during all the assessment visits (baseline and 1-, 2-, 3-, and 4-week follow-up).

\section{Secondary outcome measure}

Health related quality of life will be measured using the SF-36 questionnaire [16]. The SF-36 questionnaire is a widely used measure of general health status, consisting of eight parts: physical functioning, role-physical, bodily 
pain, general health, vitality, social functioning, roleemotional, and mental health.

\section{Safety assessments}

All subjects are to be questioned about adverse events during the treatment at each visit, and all adverse events reported will be analyzed, regardless of the investigators' assessments of causality. Safety will be assessed by complete blood cell count, erythrocyte sedimentation rate, blood chemistry, and urinalysis.

\section{Sample size}

We will estimate the required sample size for this trial based on the following calculation: $\mathrm{n}=2 \sigma^{2} \times \mathrm{f}(\alpha, \beta) /(\mu 1-\mu 2)^{2}$ [17]. First, we estimate that an absolute improvement of 4.7 (from $\mu 1$ to $\mu 2$ ) in VAS score is likely the smallest clinically-relevant difference for patients with OA. Second, we assume that standard deviation of VAS score may be $2.9(\sigma=2.9)$ at baseline [18]. Based on these assumptions, we will require 84 patients in each group to have at least a $90 \%$ power $(\beta=0.1)$ and to rule out a two-sided type I error of $5 \%(\alpha=0.05)$. The number of patients actually provides less than $80 \%$ power, assuming a withdrawal rate of $20 \%$. Therefore, we will recruit a total of 320 patients, 160 patients in each group.

\section{Statistical analysis}

The data will be collected and analyzed according to the intention-to-treat principle. Standard statistical techniques will be used to describe characteristics of patients in both groups. We will compare baseline characteristics in both groups and, if incomparability appears, we will perform a secondary analysis, adjusting for differences. The primary outcome will be compared between both groups using analysis of variance for repeated measures. All statistical analyses will be performed using SAS version 9.1 or a later version. All statistical tests will be two-sided and the level of significance will be set at 0.05 . The last-observation-carried-forward method will be used to input data with dropouts. Continuous variables will be expressed as mean $\pm \mathrm{SD}$, and categorical variables as number and percentage [19]. If adjustment for possible baseline incomparability is needed, analysis of covariance will be performed [20].

\section{Discussion}

Despite considerable study, there is no generally effective treatment for patients who suffer from OA. In some contexts, various complementary and alternative medical treatments have been administered for OA in clinical practices $[8,10,21]$. Some studies have reported the effectiveness of herbal medicine, which is the most popular form of complementary and alternative medical therapy for the treatment of OA, either used alone or concomi- tantly with usual care [6,22-26]. The quality and small sample sizes of the few trials that have been conducted have made it difficult to reach firm conclusions about these treatments. Well-designed randomized controlled trials are needed to examine the efficacy of TCM treatments for OA. The biggest advantage of the present trial is an external placebo control. The purpose of this study will be to evaluate the basic clinical efficacy and safety data for the golden plaster in the treatment of patients with knee OA.

\section{Trial status}

Patient recruitment for the trial will start from the end of 2013. Data collection will finish at the end of 2015.

\section{Abbreviations \\ HRQOL: Health-related quality of life; OA: Osteoarthritis; TCM: Traditional Chinese medicine; VAS: Visual analogue scale.}

\section{Competing interests}

The authors declare that they have no competing interests.

\section{Authors' contributions}

$J T L, D Z T, X F L, Z G Z, W B J, S T, Y J W$, and HJ contributed to the design of this study. JTL, DZT, XFL, ZGZ, WBJ, ST, YJW, and HJ contributed to the creation of the Manual of Procedures, implementation of the study protocol and acquisition of data. JTL, DZT, and XFL drafted the manuscript, and JDH modified the manuscript. All authors provided critical revision and approved the final manuscript.

\section{Acknowledgements}

This study is supported in part by the Administration of TCM of Jiangsu and Youth Health Research Project, the Program of Natural Science Foundation of China (81102604 to D.T.). We thank all the people who have been involved in our study.

\section{Author details}

'Suzhou Hospital of Traditional Chinese Medicine, 889 Wuzhongxi Road, Suzhou, Jiangsu 215009, People's Republic of China. 'Longhua Hospital, Shanghai University of Traditional Chinese Medicine, 725 Wan-Ping South Road, Shanghai 200032, PR China. ${ }^{3}$ Zhangjiagang Hospital of Traditional Chinese Medicine, Zhangjiagang, Jiangsu 215600, People's Republic of China. ${ }^{4}$ Taizhou Hospital of Traditional Chinese Medicine, Taizhou, Jiangsu 222500, People's Republic of China.

Received: 29 August 2013 Accepted: 8 November 2013 Published: 13 November 2013

\section{References}

1. Lawrence RC, Felson DT, Helmick CG, Arnold LM, Choi H, Deyo RA, Gabriel S, Hirsch R, Hochberg MC, Hunder GG, Jordan JM, Katz JN, Kremers HM, Wolfe F, National Arthritis Data Workgroup: Estimates of the prevalence of arthritis and other rheumatic conditions in the United States. Part II. Arthr Rheum 2008, 58:26-35.

2. Dillon CF, Rasch EK, Gu Q, Hirsch R: Prevalence of knee osteoarthritis in the United States: arthritis data from the third national health and nutrition examination survey 1991-94. J Rheumatol 2006, 33:2271-2279.

3. Jordan JM, Helmick CG, Renner JB, Luta G, Dragomir AD, Woodard J, Fang F, Schwartz TA, Abbate LM, Callahan LF, Kalsbeek WD, Hochberg MC: Prevalence of knee symptoms and radiographic and symptomatic knee osteoarthritis in African Americans and Caucasians: the Johnston county osteoarthritis project. J Rheumatol 2007, 34:172-180.

4. Rhon D, Zhang RW, Moskowitz RW, Nuki G, Abramson S, Altman RD, Arden $\mathrm{N}$, Bierma-Zeinstra S, Brandt KD, Croft P, Doherty M, Dougados M, Hochberg M, Hunter DJ, Kwoh K, Lohmander LS, Tugwell P: OARSI recommendations for the management of hip and knee osteoarthritis, Part II: OARSI 
evidence-based, expert consensus guidelines. Osteoarth Cartilage 2008, 16:137-162.

5. Jordan KM, Arden NK, Doherty M, Bannwarth B, Bijlsma JW, Dieppe P, Gunther K, Hauselmann H, Herrero-Beaumont G, Kaklamanis P, Lohmander S, Leeb B, Lequesne M, Mazieres B, Martin-Mola E, Pavelka K, Pendleton A, Punzi L, Serni U, Swoboda B, Verbruggen G, Zimmerman-Gorska I, Dougados M, Standing Committee for International Clinical Studies Including Therapeutic Trials ESCISIT: EULAR Recommendations 2003: an evidence based approach to the management of knee osteoarthritis: report of a task force of the standing committee for international clinical studies including therapeutic trials (ESCISIT). Ann Rheum Dis 2003, 62:1145-1155.

6. Cao Y, Zhan H, Pang J, Li F, Xu S, Gao J, Xu Z, Li G, Liu T, Guo C, Shi Y: Individually integrated traditional Chinese medicine approach in the management of knee osteoarthritis: study protocol for a randomized controlled trial. Trials 2011, 12:160

7. Macfarlane GJ, Paudyal P, Doherty M, Ernst E, Lewith G, MacPherson H, Sim J, Jones GT, on behalf of the Arthritis Research UK working group on Complementary and Alternative Therapies for the Management of the Rheumatic Diseases: A systematic review of evidence for the effectiveness of practitioner-based complementary and alternative therapies in the management of rheumatic diseases: osteoarthritis. Rheumatol (Oxford) 2012, 51:2224-2233.

8. Fransen M, McConnell S, Bell M: Therapeutic exercise for people with osteoarthritis of the hip or knee. A systematic review. J Rheumatol 2002, 29:1737-1745

9. van Baar ME, Assendelft WJ, Dekker J, Oostendorp RA, Bijlsma JW: Effectiveness of exercise therapy in patients with osteoarthritis of the hip or knee: a systematic review of randomized clinical trials. Arthr Rheum 1999, 42:1361-1369.

10. Smidt N, de Vet HC, Bouter LM, Dekker J, Arendzen JH, de Bie RA, Bierma-Zeinstra SM, Helders PJ, Keus SH, Kwakkel G, Lenssen T, Oostendorp RA, Ostelo RW, Reijman M, Terwee CB, Theunissen C, Thomas S, van Baar ME, van'tHul A, van Peppen RP, Verhagen A, van der Windt DA, Exercise Therapy Group: Effectiveness of exercise therapy: a best-evidence summary of systematic reviews. Austr J Physiother 2005, 51:71-85.

11. Roddy E, Zhang W, Doherty M: Aerobic walking or strengthening exercise for osteoarthritis of the knee? A systematic review. Ann Rheum Dis 2005, 64:544-548

12. Wang X, Cao Y, Pang J, Du J, Guo C, Liu T, Wei S, Zheng Y, Chen R, Zhan H: Traditional Chinese herbal patch for short-term management of knee osteoarthritis: a randomized, double-blind, placebo-controlled trial. Evidence-Based Comp Altern Med 2012, 2012:171706.

13. Li C, Frangione V, Rovati S, Zheng Q: Diclofenacepolamine medicated plaster in the treatment of minor soft tissue injuries: a multicenter randomized controlled trial. Curr Med Res Opin 2013, 29:1137-1146.

14. Altman R, Asch E, Bloch D, Bole G, Borenstein D, Brandt K, Christy W, Cooke TD, Greenwald R, Hochberg M, et al: Development of criteria for the classification and reporting of osteoarthritis. Classification of osteoarthritis of the knee. Diagnostic and therapeutic criteria committee of the American rheumatism association. Arthr Rheum 1986, 29:1039-1049.

15. Trijau S, Avouac J, Escalas C, Gossec L, Dougados M: Influence of flare design on symptomatic efficacy of non-steroidal anti-inflammatory drugs in osteoarthritis: a meta-analysis of randomized placebo-controlled trials. Osteoarthr Cartilage/OARS, Osteoarthr Res Soc 2010, 18:1012-1018.

16. Roos EM, Toksvig-Larsen S: Knee injury and Osteoarthritis Outcome Score (KOOS) - validation and comparison to the WOMAC in total knee replacement. Health Qual Life Outcomes 2003, 1:17.

17. Silman AJ: Clinical trials: a practical approach. $J$ R Soc Med 1984, 77:898

18. Law PP, Cheing GL: Optimal stimulation frequency of transcutaneous electrical nerve stimulation on people with knee osteoarthritis. J Rehab Med 2004, 36:220-225.

19. Kim EJ, Lim CY, Lee EY, Lee SD, Kim KS: Comparing the effects of individualized, standard, sham and no acupuncture in the treatment of knee osteoarthritis: a multicenter randomized controlled trial. Trials 2013, 14:129.

20. Yang F, Tang DZ, Cui XJ, Holz JD, Bian Q, Shi Q, Wang YJ: Classic yin and yang tonic formula for osteopenia: study protocol for a randomized controlled trial. Trials 2011, 12:187.

21. Brosseau L, Wells GA, Kenny GP, Reid R, Maetzel A, Tugwell P, Huijbregts M, McCullough C, De Angelis G, Chen L: The implementation of a community-based aerobic walking program for mild to moderate knee osteoarthritis (OA): a knowledge translation (KT) randomized controlled trial (RCT): Part I: The Uptake of the Ottawa Panel clinical practice guidelines (CPGs). BMC Public Health 2012, 12:871.

22. Chopra A, Saluja M, Tillu G, Sarmukkaddam S, Venugopalan A, Narsimulu G, Handa R, Sumantran V, Raut A, Bichile L, Joshi K, Patwardhan B: Ayurvedic medicine offers a good alternative to glucosamine and celecoxib in the treatment of symptomatic knee osteoarthritis: a randomized, double-blind, controlled equivalence drug trial. Rheumatology (Oxford) 2013, 52:1408-1417.

23. Teekachunhatean S, Kunanusorn P, Rojanasthien N, Sananpanich K, Pojchamarnwiputh S, Lhieochaiphunt S, Pruksakorn S: Chinese herbal recipe versus diclofenac in symptomatic treatment of osteoarthritis of the knee: a randomized controlled trial [ISRCTN70292892]. BMC Compl Altern Med 2004, 4:19.

24. Soeken KL: Selected CAM therapies for arthritis-related pain: the evidence from systematic reviews. Clin J Pain 2004, 20:13-18.

25. Mobasheri A: Intersection of inflammation and herbal medicine in the treatment of osteoarthritis. Curr Rheumatol Reports 2012, 14:604-616.

26. Majima T, Inoue M, Kasahara Y, Onodera T, Takahashi D, Minami A: Effect of the Japanese herbal medicine, Boiogito, on the osteoarthritis of the knee with joint effusion. Sports Med Arthros Rehab Therap Technol 2012, 4:3.

doi:10.1186/1745-6215-14-383

Cite this article as: Liu et al:: Golden plaster for pain therapy in patients with knee osteoarthritis: study protocol for a multicenter randomized, double-blind, placebo-controlled trial. Trials 2013 14:383.

\section{Submit your next manuscript to BioMed Central and take full advantage of:}

- Convenient online submission

- Thorough peer review

- No space constraints or color figure charges

- Immediate publication on acceptance

- Inclusion in PubMed, CAS, Scopus and Google Scholar

- Research which is freely available for redistribution 